\title{
MT JOHN OBSERVATORY: THE FIRST 50 YEARS
}

\author{
by J. B. Hearnshaw \\ (with ten plates)
}

Hearnshaw, J.B. 2016 (31:viii) Mt John Observatory: the first 50 years. Papers and Proceedings of the Royal Society of Tasmania 150(1): 1-8. https://doi.org/10.26749/rstpp.150.1.1 ISSN 0080-4703. Department of Physics and Astronomy, University of Canterbury, Christchurch, New Zealand. Email: john.hearnshaw@canterbury.ac.nz

Mt John Observatory at Lake Tekapo is New Zealand's only professional research observatory. It was founded in 1965 as a joint institution of the universities of Pennsylvania and Canterbury, though the American participation disappeared after 1980. Some of the successes of the past 50 years and also the numerous problems that arose are described here. Four telescopes have been established at Mt John, including the 1.8-m MOA telescope in 2004, dedicated to microlensing. Astro-tourism has flourished in the past decade.

Key Words: observational astronomy, NewZealand, optical telescopes, stellar spectroscopy, gravitational microlensing, astro-tourism.

\section{INTRODUCTION}

The Mt John Observatory at Lake Tekapo in the central South Island of New Zealand was founded in 1965 by the universities of Canterbury and Pennsylvania, after a site survey initiated in 1962. The key players were Prof. F. Bradshaw Wood at Pennsylvania and Frank Bateson, a New Zealand amateur astronomer who conducted the site survey and became the first astronomer-in-charge at Mt John.

The motivation for establishing the observatory was to give Pennsylvania astronomers access to the southern sky, especially for variable and binary star observations. At this time astronomy was just starting as a discipline for teaching and research at Canterbury, but local support for the new observatory to provide the necessary logistics was seen as essential. Pennsylvania offered and sent a large $45-\mathrm{cm}$ aperture refractor telescope dating from the $1890 \mathrm{~s}$, known as the Brashear telescope. It was formerly installed at the Flower Observatory near Philadelphia. This was to be the major telescope at Mt John and it arrived in 1963 after refurbishment. Fortunately it was never installed, as it represented outmoded technology and would have required a very large dome and building.
Bateson obtained a large lottery grant of $£ 30000$ (about $\$ 1.2$ million in today's money) to establish the observatory, and four buildings were erected using these funds (see pl. 1). But the money ran out before the Brashear telescope could be installed. There is some irony in the fact that $\mathrm{Mt}$ John depended on the Brashear telescope as the primary telescope to justify its foundation, yet the instrument has now spent some 50 years in storage without making one observation in New Zealand. It will soon be installed near Mt John for public outreach.

\section{SOUTHERN HEMISPHERE OBSERVATORIES IN THE 1960s}

In 1960 there were only 10 astronomical observatories south of the equator, compared to 88 in the northern hemisphere (Blanco 1993). Only a few of those in the south were engaged in astrophysics, rather than positional astronomy, and these included Mt Stromlo near Canberra, and the Royal Observatory in Cape Town.

The 1960s and 1970s saw a huge expansion of new observatories, including the Cerro Tololo Interamerican

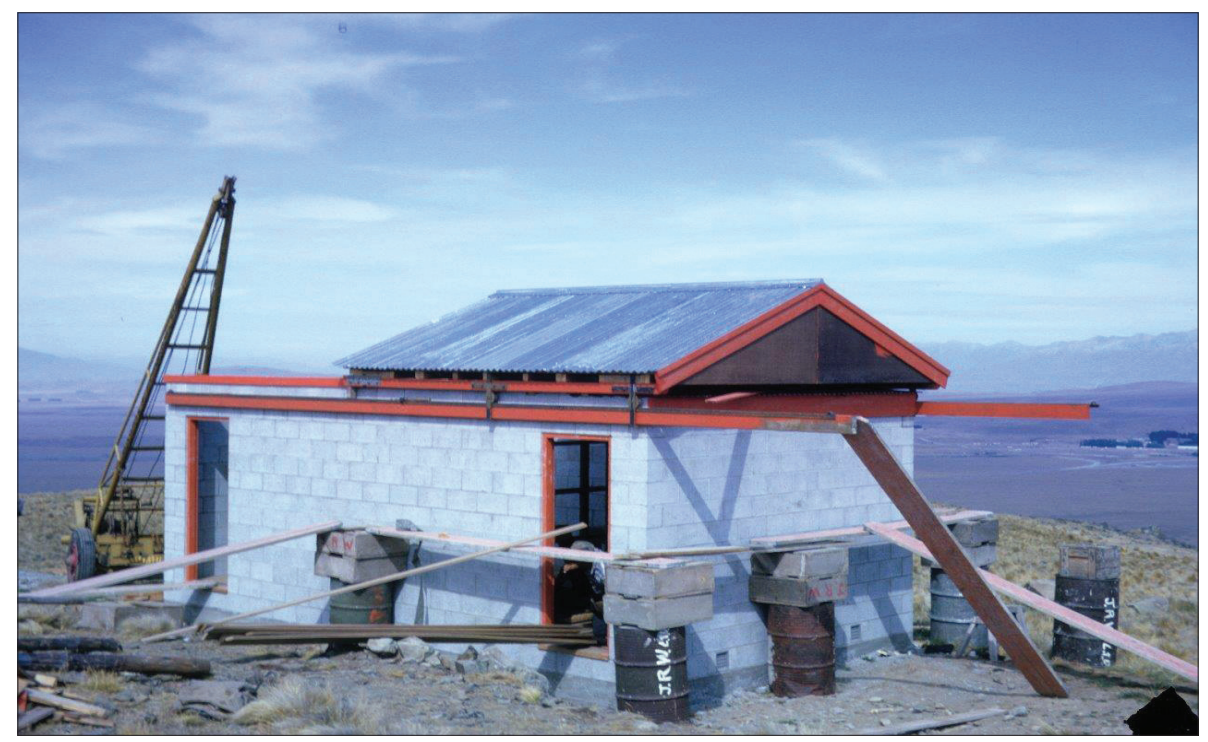

PLATE 1 - The astrograph building on Mt John under construction in 1964. 


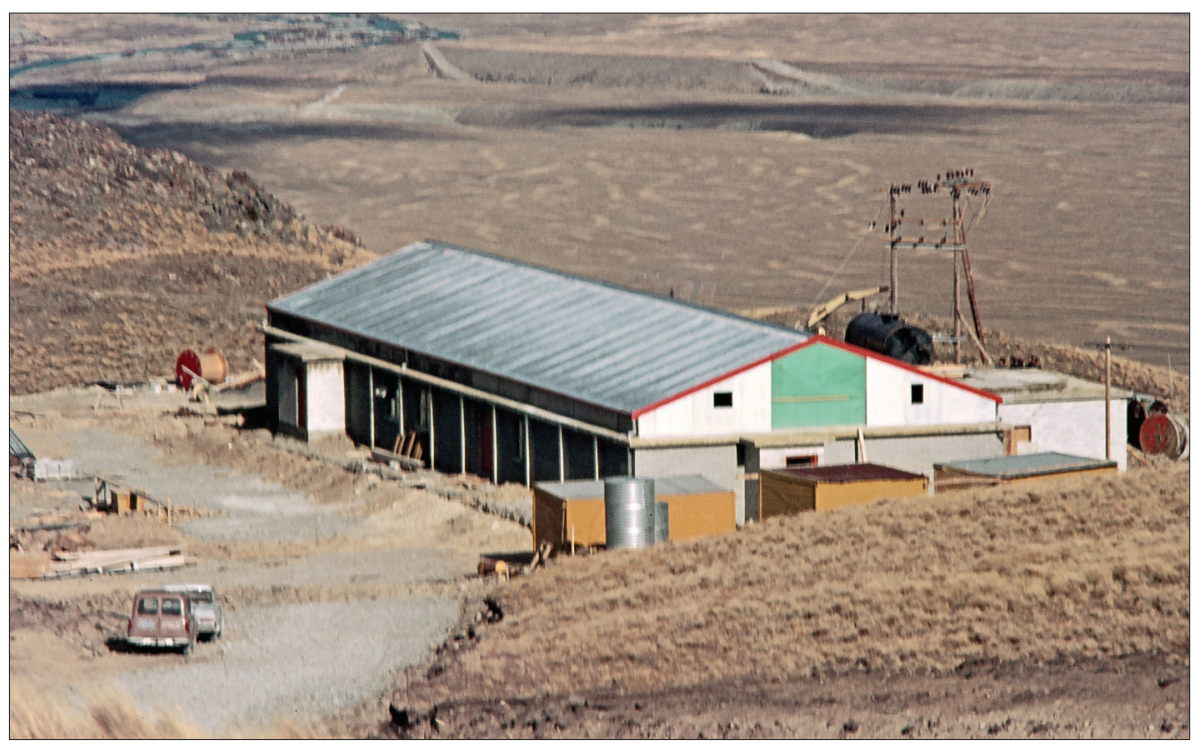

PLATE 2 - The US satellite tracking station under construction in 1968.

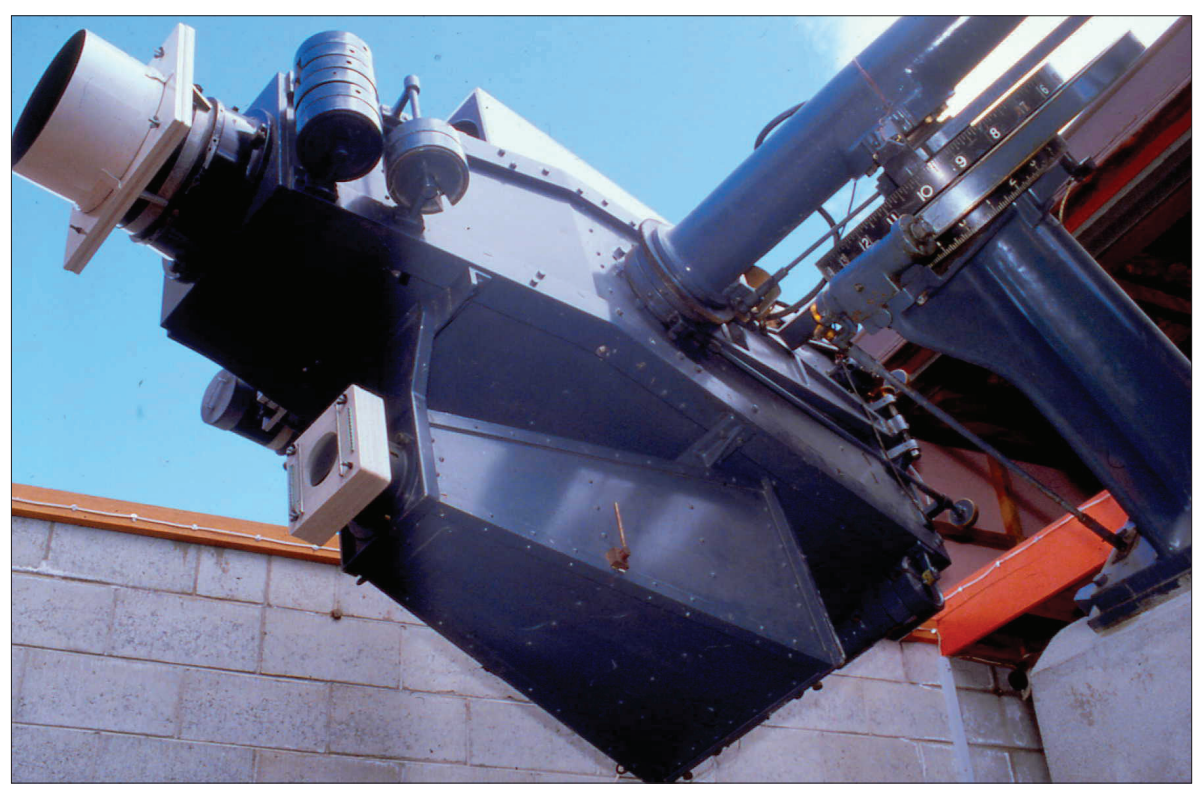

PLATE 3 - The triple astrograph on Mt John, used for the Canterbury Sky Atlas, 1972.

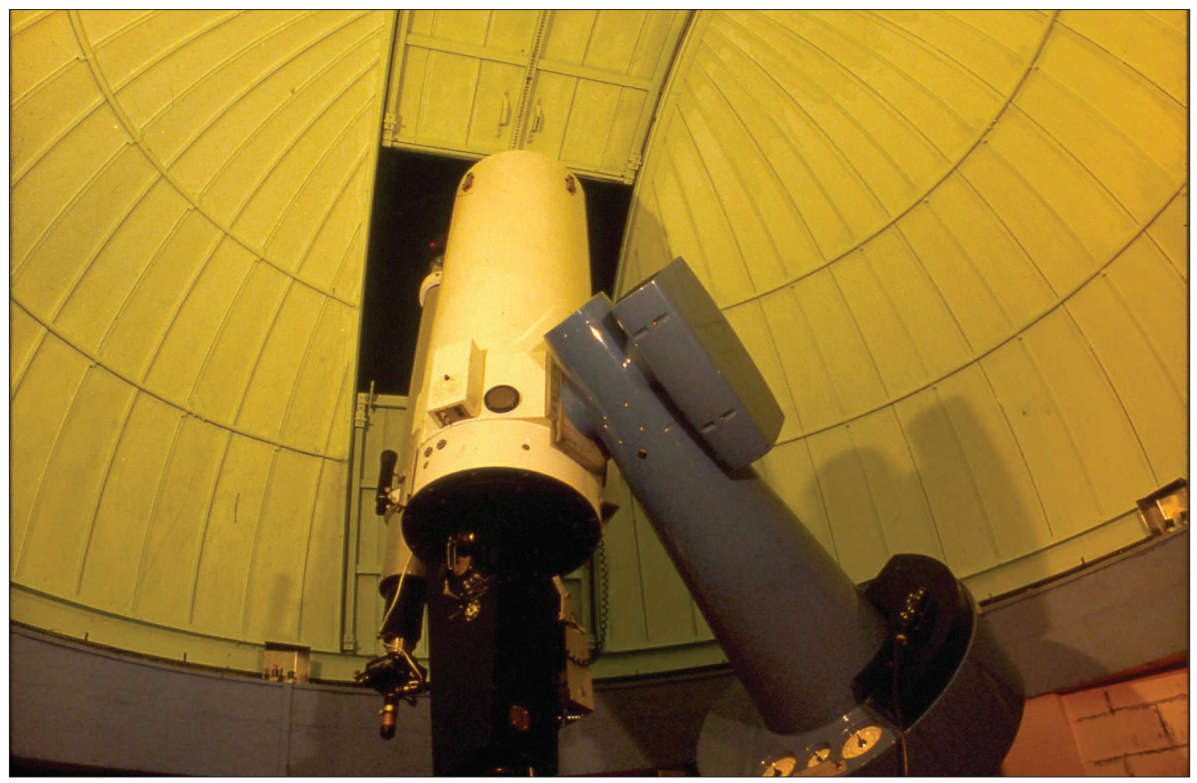

PLATE 4 - The B\&C $60-\mathrm{cm}$ telescope with the Cassegrain échelle spectrograph, in 1977. 
Observatory (Chile, 1964), the European Southern Observatory (Chile, 1969), las Campanas Observatory (Chile, 1969) and the Anglo-Australian Observatory (New South Wales, 1974). Mt John was part of this thrust to develop new facilities in the south. However, it was supported by just one partner university in the northern hemisphere, so could not compete with the much larger multinational observatories being developed elsewhere, especially in Chile. At this time research in astrophysics was poorly developed in New Zealand universities, and the country was 40 years behind Australia in teaching courses, taking on graduate students and providing observational facilities.

\section{THE US AIR FORCE COMES TO MT JOHN}

A significant event in the history of $\mathrm{Mt} \mathrm{John} \mathrm{was} \mathrm{the} \mathrm{arrival}$ of the US Air Defense Command in 1968, just three years after the establishment of the observatory. A satellite tracking station was constructed near the summit of Mt John, just to the south of the observatory, and on land leased by the University ofCanterbury from the Crown. A 476-square metre building (pl. 2) was constructed in 1968, which housed a Baker-Nunn camera, together with offices, accommodation (six bedrooms), a workshop, dining room, kitchen, a communications centre and garages. The whole facility was staffed by American military personnel in uniform, with the approval of the University of Canterbury council, the New Zealand Department of External Affairs (now Ministry of Foreign Affairs and Trade) and the strong support of the University of Pennsylvania, with the claim that "the work was unclassified and the results would be freely available" (Hearnshaw \& Gilmore 2015).

The presence of the US Air Force did nevertheless bring some material benefits: a sealed road, a crash barrier on the road and a pumped water supply from the lake, which was shared by the observatory from 1971. Before then Mt John had to rely on collected rainwater as its sole supply. Yet it was an extraordinary relationship which would be unconscionable by the standards of today. It was not surprising therefore that in March 1972 a major demonstration (led by the late Owen Wilkes) of some 300 students and activists took place on $\mathrm{Mt}$ John against the military presence on university land. Thanks to advance intelligence, some 60 police officers were present; nevertheless, considerable damage was done, mainly to observatory facilities and to the road. The tracking station itself was largely unscathed (Hearnshaw \& Gilmore 2015).

The upshot of the demonstration was that the military personnel were replaced by civilians in 1972, and this enabled satellite tracking to continue until 1983 when the US tracking station was closed. This whole episode had several lasting consequences for the observatory, some positive, some negative. In the public's mind, the distinction between the two separate facilities on Mt John was generally not made, and the university observatory was generally associated with a secret military operation. Also, a small block of land around the satellite tracking station was removed from the university's lease and leased directly from the Crown to the US Government. As a result, when the tracking station eventually closed, the university had to re-acquire that land and take over the vacated building at great expense, paying an exorbitant rental to the New Zealand government for its use. On the positive side, the American facility became the building to house Canterbury's new 1-m McLellan telescope in 1986 and the bedrooms, kitchen and dining room became the centre of the observatory's new accommodation facility from that time (Hearnshaw \& Gilmore 2015).

\section{EARLY RESEARCH WORK}

The first telescopes at Mt John were two small telescopes owned by Frank Bateson (a 40-cm reflector and a 20-cm refractor), the 45-cm Brashear refractor from Pennsylvania, which arrived in 1963 but was never installed, and three astrographic cameras (apertures 10, 15 and $25 \mathrm{~cm}$ ) for wide-field photographic survey work (pl. 3). The astrographs were originally at the Cook Observatory of the University of Pennsylvania. They were used in the years 1966-1972 at Mt John to produce the Canterbury Sky Atlas (Doughty et al. 1972), a 16thmagnitude atlas of the southern sky to complement the Lick Observatory Atlas (Shane 1958, Lick Observatory Sky Atlas 1965). The atlas was recorded on large-format glass plates using a blue-sensitive photographic emulsion. It was one of the first large projects undertaken at Mt John.

Apart from the work on the Canterbury Sky Survey, the main activity at the observatory was the observation of the light curves of southern eclipsing binary stars using a photoelectric photometer. Such observations were the principal interest of Frank Bradshaw Wood and his colleagues (notably William Protheroe and Bob Koch) at Pennsylvania. At first this work was undertaken using Bateson's own 40-cm reflector: from 1970 a 61-cm telescope was installed by Pennsylvania to continue this work. Bateson had meanwhile resigned as astronomer-in-charge on $\mathrm{Mt}$ John in 1969 and removed his own telescopes, after the University of Canterbury declined to purchase them at what was considered to be an exorbitant price.

The University of Pennsylvania sent a number of graduate students to Mt John who used the telescopes to observe and analyse eclipsing binary light curves. Typically two students were sent for deployments in New Zealand for a year. The weather was frequently unfavourable for good quality photometric work, and the poor performance of the two reflecting telescopes was an additional hindrance.

\section{NEW TELESCOPES}

It was only in the late 1960 s that the University of Canterbury became seriously interested in astronomy and started hiring lecturers to teach undergraduate courses in astronomy and to supervise graduate students. At the same time, the university made a commitment to Mt John, when in 1975 a second 61-cm reflector was purchased from Boller \& Chivens (B\&C) in the US (pl. 4). It, too, was used for photometry and from 1976 a medium-resolution spectrograph was installed. Ayear later, Canterbury constructed a high-resolution Cassegrain échelle spectrograph for the B\&C telescope, enabling detailed analyses of the spectra of bright stars (Hearnshaw 1977, 1978). This type of work proved to be ideal for Mt John, given the many nights which are not fully photometric.

By 1980 it was clear that a new larger telescope was a high priority for Mt John. Given that the departmental workshops employed technicians skilled in mechanical design and construction, in electronics and computer control and in optics, a new project to design and build a 1-m telescope was initiated. Funding came from the university, the Department of Physics, from the lottery and 

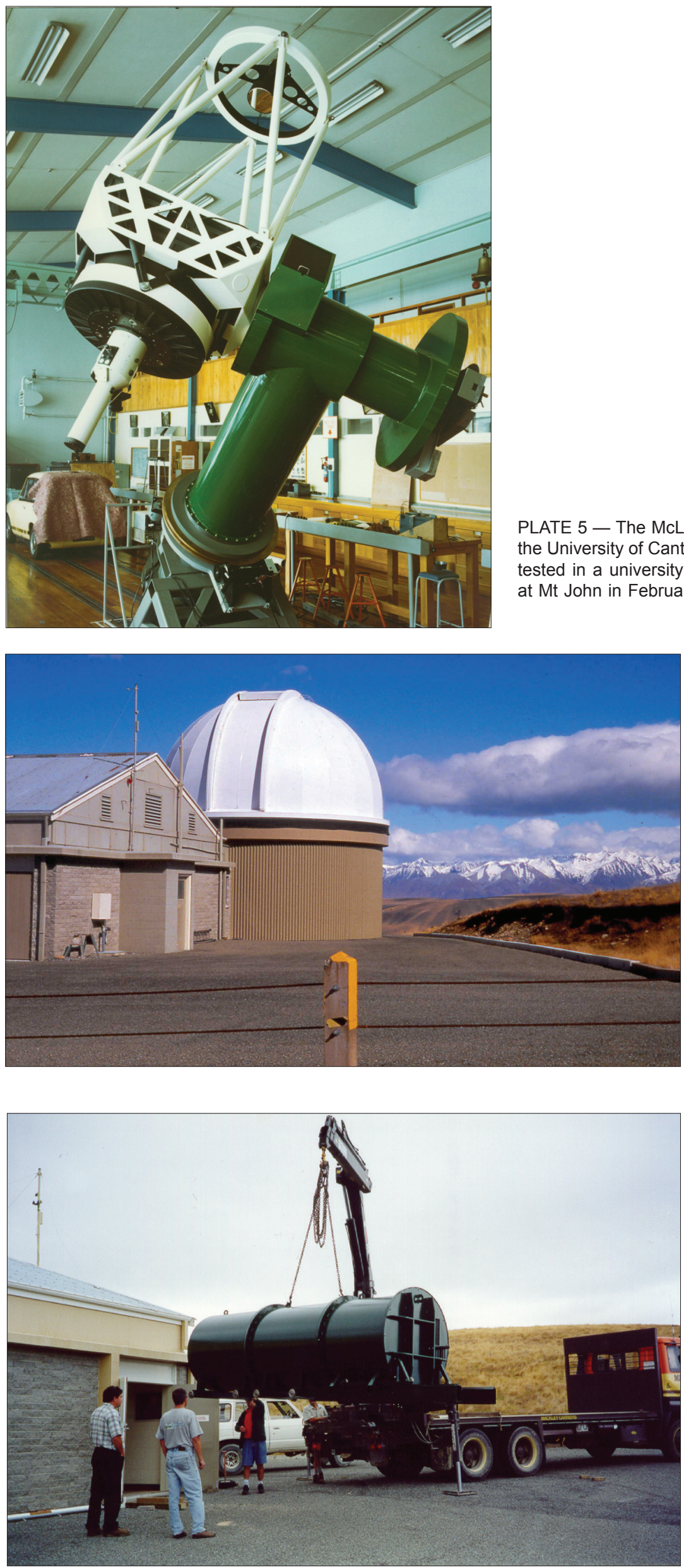

PLATE 5 - The McLellan 1-m telescope was designed and built at the University of Canterbury in the early 1980s. It is seen here being tested in a university workshop in late 1985 prior to its installation at Mt John in February 1986.
PLATE 6 - The dome of the McLellan 1-m telescope with the Sothern Alps in the background.
PLATE 7 - The vacuum tank of the HERCULES high-resolution spectrograph arrives at Mt John for installation at the 1-m telescope in February 2001. 
from a public appeal for funds. Construction commenced in 1981 and the 1-m Zerodur mirror blank was figured in the department by Garry Nankivell from the Department of Scientific and Industrial Research (DSIR) Physics and Engineering Laboratory. The telescope has a Cassegrain Dall-Kirkham optical arrangement with focal ratios of either $\mathrm{f} / 13.5$ or $\mathrm{f} / 8$, depending on the choice of secondary mirror. The $\mathrm{f} / 8$ configuration with field-correcting lenses gave good stellar images over a $1.7^{\circ}$ field. The mechanical design and construction were undertaken by university technician, Graeme Kershaw, and the whole project was completed by the end of 1985, with the telescope tested on campus (pl. 5). Installation at Mt John followed in February 1986, in the former US satellite tracking station building after major refurbishment. Part of the overall telescope project was the construction of the 8-m diameter wooden dome (pl. 6) and the construction of a vacuum aluminising plant for the 1-m mirror.

The Mt John 1-m telescope was named the McLellan telescope after the former head of the Department of Physics, Prof. A.G. McLellan. It has been the main workhorse for Mt John for the past 30 years. It has been used primarily for high-resolution stellar spectroscopy, but also for CCD photometry, medium-resolution spectroscopy and astrometry.

\section{THE HERCULES FIBRE-FED VACUUM ÉCHELLE SPECTROGRAPH}

A new fibre-fed échelle spectrograph was designed and built in the Department of Physics and Astronomy at Canterbury from 1998-2001. Once again, Graeme Kershaw undertook the mechanical work and Garry Nankivell (then retired and operating from his optical workshop at home) undertook most of the optics. Industrial Research Ltd (formerly DSIR Physics and Engineering Lab) made the large BK7 glass prism for cross-dispersion of the échelle orders. The new instrument was named HERCULES, for the High Efficiency and Resolution Canterbury University Large Echelle Spectrograph. HERCULES was the world's first, fibre-fed, vacuum, échelle spectrograph. The large vacuum tank (4.5 $\mathrm{m}$ long, $1.2 \mathrm{~m}$ in diameter) enclosed a vacuum at about 3 torr with all the spectrograph components except the $4 \mathrm{k} \times$ $4 \mathrm{k}$ CCD detector being inside the vacuum, and with no moving parts (Hearnshaw et al. 2002).

The main components of HERCULES are a $20-\mathrm{cm}$ parabolic collimator, an R2 échelle grating, $20 \mathrm{~cm} \times 40 \mathrm{~cm}$ in dimensions, a BK7 cross-dispersion prism weighing $23 \mathrm{~kg}$ (probably the largest optical prism made in New Zealand), a folded Schmidt camera comprising a Schmidt corrector plate, a 50-cm spherical camera and a fold flat perforated with a central off-axis hole. A small field-flattening lens immediately in front of the CCD camera also serves as a window between the vacuum and external air.

HERCULES was designed especially for the precise measurement of stellar radial velocities, which are crucial for the detection of extrasolar planets by the Doppler technique. For solar-type stars, radial-velocity precisions of about $3 \mathrm{~m} / \mathrm{s}$ have been demonstrated from a single spectrum at a resolving power of 70 000. HERCULES was fed by a $25-\mathrm{m}$-long optical fibre taking light from the $\mathrm{f} / 13.5$ Cassegrain focus to a heavily insulated and thermally controlled spectrograph room, which was adapted from the old tracking-station photographic dark room. This room, controlled to about $\pm 0.1^{\circ} \mathrm{C}$, provided a very stable environment for the spectrograph operations.

HERCULES went into operation in April 2001 and has been by far the most used instrument on the 1-m telescope since that time (pl. 7). It has been used for studies of binary and variable stars, searches for extrasolar planets, studies of the chemical composition of stars, studies of stellar rotation and of asteroseismology.

\section{THE MOA PROJECT AND 1.8-m MOA TELESCOPE}

The MOA project at Mt John was started in 1994 as a collaboration between Auckland, Nagoya and Canterbury universities, with the support of several other institutions in Japan and New Zealand. MOA stands for Microlensing Observations in Astrophysics, and the project aimed to detect extrasolar planets or other dark massive objects in our Galaxy by making photometric observations of the light of stars which were briefly amplified by the gravitational effect of an intermediate lens star or object precisely aligned along the line-of-sight to the source. The amplification effect is detectable when brief alignments of source and lens occur to within about a milli-arc second or better. Microlensing is an effect predicted by Einstein in 1937, though he estimated that such precise alignments would be so rare, they were unlikely ever to be observed (Einstein 1937). The key people in MOA were Professors P. Yock (Auckland) and Y. Muraki (Nagoya), with participation from I. Bond (Massey University at Albany), D. Sullivan (Victoria University Wellington) and J. Hearnshaw (Canterbury).

Following the first detections of microlensing from Australia in 1990, the Mt John MOA project started a few years later, using at first the B\&C telescope, modified for a wider field of view. Nagoya University provided a large multi-chip CCD camera. The aim was to search for new microlensing events in crowded star fields in the galactic bulge where about one star in a million can be expected to be undergoing microlensing as a result of the passage of a lens star through the line of sight. The detection of extrasolar planets was a primary goal. A secondary aim in the early years was to search for massive compact lensing objects in the galactic halo, possibly black holes which might account for missing mass in our Galaxy, by observing star fields towards the Magellanic Clouds. The absence of such massive objects in the galactic halo was soon demonstrated by MOA and others. On the other hand, the galactic bulge proved to be a fertile hunting ground for microlensing events, with about 50 or more events being detected in the early years of the project. A decade later, the number of events alerted by the MOA team each year in the galactic bulge had grown to well over 600 .

It took MOA about eight years before the first definitive case of an extrasolar planet being discovered from the microlensing light curve (Bond et al. 2004). This was for the event MOA-2003-BLG-53, which was jointly discovered with the OGLE (Optical Gravitational Lensing Experiment) team in Chile. The analysis of the light curve indicated the presence of a 1.5 Jupiter-mass gas giant orbiting a low-mass dwarf star (the lens) at a distance of about three astronomical units.

In July 2002, Y. Muraki at Nagoya University obtained a large grant from the Japanese Ministry of Education and Science for the construction of a $1.8-\mathrm{m}$ alt-az wide-field 

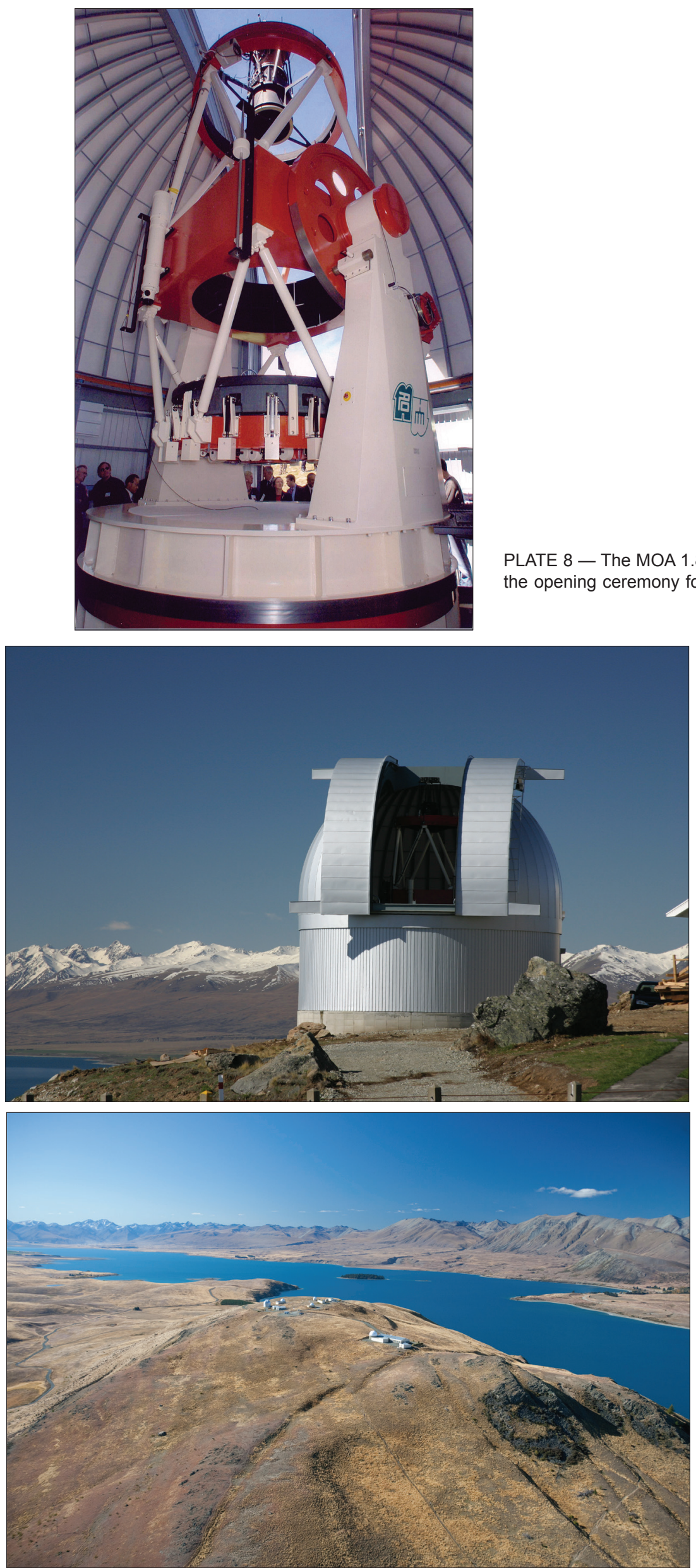

PLATE 8 - The MOA 1.8-m telescope at Mt John seen at the time of the opening ceremony for the telescope in December 2004.
PLATE 9 - The MOA dome at Mt John with the Two Thumb range seen in the background.
PLATE 10 - Mt John Observatory seen from the air with Lake Tekapo and the Two Thumb range beyond the lake. The McLellan telescope dome is nearest to the camera, the MOA dome is farthest left. The outstanding panorama seen from the observatory and the exceptionally clear air have attracted many thousands of astro-tourists to Mt John in the past 10 years. 
imaging telescope to be installed at Mt John for the MOA project. The telescope was constructed by the Nishimura telescope company in Kyoto and installed at Mt John in late 2004 (pl. 8). The optical design was by A. Rakich of KiwiStar optics in Wellington; KiwiStar also made the four prime-focus corrector lenses. The telescope is equipped with a 10-chip CCD camera with 80 million pixels, placed at the telescope's prime focus.

The $1.8-\mathrm{m}$ MOA telescope dedicated to microlensing has led to a large increase in the number of microlensing alerts in the galactic bulge and to the number of extrasolar planets detected by microlensing. Most of the planetary discoveries have been joint efforts with other microlensing groups observing the same event from a range of longitudes, as a full analysis requires constant 24-hour monitoring of an event over several weeks. By late 2014 the MOA project had been operating for two decades, and for almost one decade with the 1.8-m telescope (pl. 9). Twenty-nine extrasolar planets had been discovered by microlensing, with MOA contributing data to all these discoveries. Some of these were only a few Earth-masses, so are presumably rocky objects, in or close to their habitable zones where water can be liquid.

One of the most important results from the MOA project, and perhaps the most significant in Mt John's 50-year history, came in 2011, with the discovery in the Galaxy of free-floating planets, those not attached to any star (Sumi et al. 2011). Probably they were ejected from stellar orbits by another massive orbiting planet or by a nearby passing star. The original paper reported ten such objects from observations of short-duration microlensing events, lasting a few hours to about two days, indicating microlensing by gas giant planets. Statistical estimates of the number of free-floating planets in the Galaxy indicate a possible population of several hundred billion, possibly even greater than the number of stars.

\section{MT JOHN AND ASTRO-TOURISM}

In 2004 the University of Canterbury gave exclusive tourism rights to the newly-founded company Earth \& Sky Ltd at Tekapo, to take visitors to Mt John Observatory. In 2007 Earth \& Sky built the Astrocafe on Mt John, comprising a glass atrium around an older and redundant building housing a sky camera. Visitors are able to visit the observatory during the day and go on guided starwatching tours at night. The day visitors come to admire the outstanding view over the Mackenzie Basin and Lake Tekapo (pl. 10), to visit the Astrocafe and some go on guided tours to see the telescopes.

Opening up the observatory to astro-tourists has been almost too successful, with visitor numbers straining the observatory's resources. An estimate of over 120000 visitors a year is a conservative one based on the count of vehicles driving to the summit of Mt John during the day and on ticket sales for guided night visits. Thousands more walk to the observatory from Tekapo village, but their numbers are not precisely known. In the past decade, Mt John has become a dual-role observatory, catering for both astronomical research and public outreach. Well over a million people have set foot on Mt John in the past decade.

In 2012 an application was made to establish an International Dark Sky Reserve in the Mackenzie Basin and the Aoraki/Mt Cook National Park. The application was made to the International Dark-Sky Association headquartered in Tucson, Arizona, and the outcome was the successful accreditation of the reserve with gold status (the highest awarded). It was the first such reserve in the southern hemisphere, just the third in the world to be inscribed, the largest in the world at 4367 square kilometres and the first to be accorded gold tier status. The reserve built on the lighting ordinances that had been in place since 1981 to protect Mt John from light pollution, as well as the success of Earth \& Sky and the scientific reputation of Mt John. The result has been a further large increase in astro-tourism at Lake Tekapo. Mt John is now one of New Zealand's premier tourist attractions, especially for tourists from China and Japan who are coming in ever greater numbers to see a truly pristine unpolluted night sky, which many have never before seen from the large urban centres of their homelands.

\section{CONCLUDING REMARKS AND SUMMARY}

Mt John Observatory is now 50 years old. In this time, its reputation for scientific research has grown, based on about 1000 scientific papers in the astronomical literature that have made use of observations at Mt John. By the end of 2014, 82 University of Canterbury graduate students had earned MSc or PhD degrees using Mt John data. In addition, 93 graduate students from other New Zealand universities or from overseas universities (notably, but not only, from the US and Japan) have written theses based on Mt John data. Most of these students have come in person to collect their observations.

Mt John has capitalised on its unique geographical location, as the world's southernmost observatory, and as the only major observatory in its longitude zone. Both latitude and longitude confer distinct advantages for astronomical observing: the latitude because some far southern objects (e.g., the Magellanic Clouds) are circumpolar and visible all year; the longitude because it enables collaborative multi-site observations where time coverage is critical.

Mt John has been able to make a number of key discoveries in its 50-year existence. They include a supernova, a nakedeye nova, three comets, many new asteroids, free-floating planets, one planet in a binary system by the Doppler technique, and two optical transients from gamma-ray bursters. In fact the observatory's main output has been a large number of results on variable stars, binary stars, asteroseismology, minor planet astrometry and gravitational microlensing, too numerous to mention.

In 2015, J. Hearnshaw and A. Gilmore wrote a book entitled Mt John - the first 50 years: a celebration of half a century of optical astronomy at the University of Canterbury (Hearnshaw \& Gilmore 2015). This richly-illustrated book for the lay reader was published by Canterbury University Press. It gives the full story of the observatory's development and history, including the many personality battles, funding crises and scientific triumphs along the way which characterised the observatory's development.

Today, the battle to continue a viable scientific observatory in New Zealand continues. After the 2011 earthquakes in Christchurch, the University of Canterbury came under extreme financial pressure. Mt John was not directly affected, being $200 \mathrm{~km}$ from the quake epicentres, but the financial fallout has squeezed many university facilities. (The scientific staffing levels at Mt John have fallen below a minimum critical mass. The result is a continuing struggle to ensure 
that the observatory remains viable as it enters its second half century.)

\section{REFERENCES}

Blanco, V. 1993: Brief history of Cerro Tololo Interamerican Observatory, www.ctio.noao.edu/noao/content/ctiohistory, accessed 26 April 2016.

Bond, I.A., Udalski, A., Jaroszyński, M., Rattenbury, N.J., Paczyński, B., Soszyński, I., Wyrzykowski, L., Szymański, M.K., Kubiak, M., Szewczyk, O. and 23 co-authors 2004: OGLE 2003-BLG-235/MOA 2003BLG-53: A Planetary Microlensing Event Astrophysical Journal Letters 606: L155-L158.

Doughty, N.A., Shane, C.D. \& Wood, F.B. 1972: The Mt John University Observatory Photographic Sky Survey and Canterbury Sky Atlas (Australis). University of Canterbury, Christchurch: 15 pp. plus 46 photographic plates.

Einstein, A. 1937: Lens-Like Action of a Star by the Deviation of Light in the Gravitational Field, Science 84: 506-507.

Hearnshaw, J.B. \& Gilmore, A.C. 2015: Mt John - the first 50 years: a celebration of half a century of optical astronomy at the University of Canterbury. University of Canterbury Press, Christchurch: 216 pp.
Hearnshaw, J.B. 1977: The Cassegrain Echelle Spectrograph at Mt John Observatory, Proceedings of the Astronomical Society of Australia 3: 102-103.

Hearnshaw, J.B. 1978: An echelle spectrograph, Sky and Telescope 56: $6-8$.

Hearnshaw, J.B., Barnes, S.I., Kershaw, G.M., Frost, N., Graham, G., Ritchie, R. \& Nankivell, G.R. 2002: The Hercules Échelle Spectrograph at Mt John, Experimental Astronomy 13: 59-76.

Lick Observatory Sky Atlas 1965: Lick Observatory, University of California, Santa Cruz, CA, USA.

Shane, C.D. 1958: A new sky atlas, Publications of the Astronomical Society of the Pacific 70: 609-610.

Sumi, T., Kamiya, K., Bennett, D.P., Bond, I.A., Abe, F., Botzler, C.S., Fukui, A., Furusawa, K., Hearnshaw, J.B. Itow, Y. and 29 co-authors 2011: Unbound or distant planetary mass population detected by gravitational microlensing, Nature 473: 349-352.

(Accepted 3 May 2016) 